La programmation de la toute

première expérience numérique, dite de Fermi-Pasta-Ulam (FPU), a été effectuée par une jeune femme nommée Mary Tsingou dont le nom a été

malencontreusement oublié.

Après plusieurs années

de recherches infructueuses,

il m'a été possible de retrouver

sa trace tout près du lieu

de ses premiers travaux.

\section{Cette simulation numérique}

est d'une importance capitale

en physique non linéaire.

Il est temps de faire réapparaitre le nom de Mary Tsingou,

qui a été écrit à l'encre

sympathique sur l'article

fondateur : reconnaissons

sa contribution et parlons

désormais du problème

de Fermi-Pasta-Ulam-Tsingou

(FPUT).

\title{
La (dis-)simulation FPU : une Femme Physicienne Underground
}

\section{Mary Tsingou et l'histoire de la première expérience numérique}

Thierry Dauxois (Thierry.Dauxois@ens-lyon.fr)

Laboratoire de Physique de l'ENS de Lyon (UMR5672 CNRS, Université Claude Bernard Lyon 1, École normale supérieure de Lyon), 69342 Lyon Cedex 07

\section{Le problème FPU}

Le problème de Fermi-Pasta-Ulam (FPU) [1], d'abord décrit dans un rapport classifié de Los Alamos en mai 1955, est souvent considéré comme le point de départ d'un nouveau domaine, la physique non linéaire ; mais c'est aussi la toute première expérience numérique. L’idée était de simuler l'analogue unidimensionnel d'atomes dans un cristal : une longue chaine de masses reliées par des ressorts qui obéissent à la loi de Hooke (une interaction linéaire) à laquelle on rajoute un faible terme non linéaire. Une interaction purement linéaire garantirait que l'énergie introduite dans un seul mode de vibration de Fourier reste toujours dans ce mode ; le terme non linéaire permet en revanche le transfert d'énergie entre les modes. Dans certaines conditions, le système faiblement non linéaire présente cependant un comportement surprenant : il n'évolue pas vers l'équipartition de l'énergie prévue par la physique statistique mais retourne périodiquement vers la situation de départ (fig. 1). Ce résultat tout à fait remarquable, connu sous le nom de paradoxe de FPU, montre que la non-linéarité ne suffit pas à garantir l'équipartition de l'énergie.

Dans les années 1960, à la recherche de la solution du paradoxe de FPU, Norman Zabusky et Martin Kruskal ont examiné le problème dans l'espace réel plutôt que dans l'espace de Fourier [2]. Cela leur a permis d'expliquer le comportement périodique grâce à la dynamique d'excitations non linéaires et localisées spatialement, que
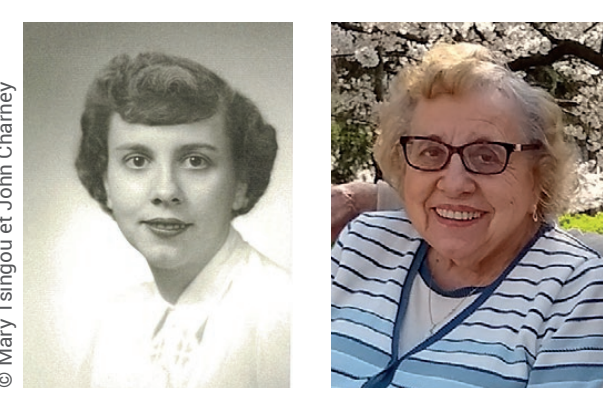

Mary Tsingou en 1955 et en 2019.

l'on connait de nos jours sous le nom de "solitons ". Ces ondes localisées, ou solitaires, auxquelles on peut associer des propriétés de particules (d'où le suffixe "-on » qui a justement été proposé dans cet article) ont de nombreuses applications physiques et constituent aujourd'hui un champ d'étude à part entière. Il couvre non seulement les tsunamis (un mot d'origine japonaise dont la signification, "vague dans le port ", traduit très bien son aptitude à détruire) mais aussi la capacité de transmettre de l'information à très haut débit dans des fibres optiques, en passant par de nombreuses autres applications physiques [3] Une autre ligne de recherche issue du paradoxe de FPU, développée parallèlement aux travaux sur les solitons, s'est plus particulièrement focalisée sur la dynamique du mode de Fourier de départ. En particulier, avec la découverte du théorème de Kolmogorov-Arnold-Moser, il a été prouvé qu'en général les orbites dans l'espace des phases de systèmes hamiltoniens intégrables 
restent quasi périodiques lorsqu'ils ne sont que légèrement perturbés. En revanche, si la perturbation est trop forte, la récurrence est détruite et l'équipartition de l'énergie s'établit rapidement [4].

Le problème de FPU est donc d'une importance centrale dans le domaine des solitons ainsi que dans celui du chaos, et est également intimement lié à la physique statistique hors équilibre [5] (voir l'encadré, p. 29). Encore aujourd'hui, de nombreux articles, séminaires et conférences sont dans cette lignée. Mais, malgré toute l'attention portée au problème de FPU et à son histoire, un point intrigant n'a guère été mentionné.

\section{Le mystère des signatures}

La première page du rapport sur le travail déposé en 1955 à Los Alamos indique : "Report written by Fermi, Pasta, and Ulam. Work done by Fermi, Pasta, Ulam, and Tsingou."

Cette remarque, selon laquelle Mary Tsingou a participé à l'étude numérique mais n'est pas auteure du rapport, a déconcerté les scientifiques qui ont lu ce document. Programmer un ordinateur dans les années 1950 n'était pas une tâche triviale. Pourquoi sa contribution n'a-t-elle reçu qu'une note de deux lignes?
Ceux qui approfondissent la littérature sur FPU ont généralement lu un article publié en 1972 de James Tuck et M. T. Menzel [6]. Une lecture attentive de l'introduction révèle que Menzel a été impliqué dans le codage du problème original, mais personne de ce nom n'est mentionné dans le rapport de Los Alamos. Comment résoudre ce paradoxe?

La solution est que dans le nom M. T. Menzel, $M$ est pour Mary et T pour Tsingou. Il n'y a donc pas de paradoxe, c'est la même personne, utilisant son nom de femme mariée ! Intrigué par cet élément, je me suis demandé alors ce que cette femme était devenue et j'ai posé des questions, longtemps restées sans réponse. Un jour, j'eu l'idée d'utiliser tout simplement Web of Science (qui a donc bien une utilité !) qui révéla que M.T. Menzel avait publié avec un certain J.L. Gammel. Coup de chance, au cours de ma thèse, j'avais logé plusieurs mois à Los Alamos chez Tinka Gammel, la fille de ce dernier. À peine avais-je évoqué cette histoire à ma logeuse (que je savais concernée par la cause des femmes), qu'elle fit son possible pour m'aider ; et nous avons découvert que son propre père, qui travaillait encore dans ce prestigieux laboratoire, avait effectivement travaillé avec M. Menzel, née Tsingou. Il l'avait perdu de vue, mais sa trace a pu être retrouvée. expérience numérique. [...] Son résultat, tout à fait remarquable, montre que la non-linéarité ne suffit pas à garantir l'équipartition de l'énergie."

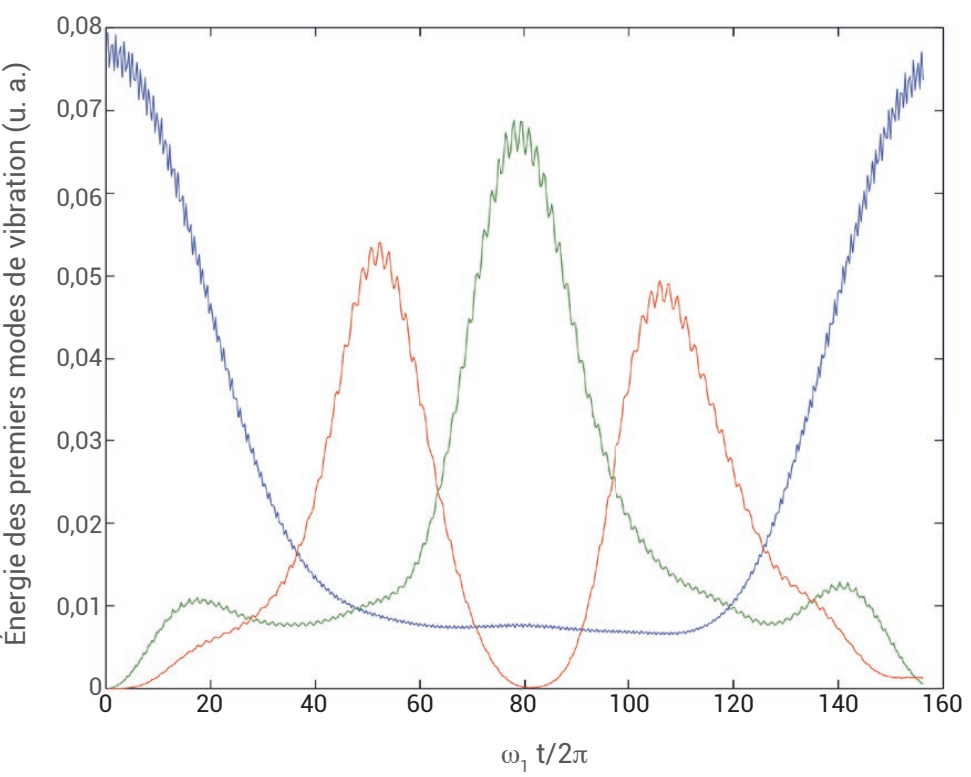

1. La récurrence FPU. Évolution de l'énergie totale (cinétique + potentielle) des trois premiers modes linéaires dans l'expérience numérique de FPU. Initialement, seul le mode de plus grande longueur d'onde est excité (ligne bleue). Après un transfert vers les modes 2 (vert), 3 (rouge) ..., l'énergie revient presque complètement vers le premier mode : cette récurrence fut une surprise totale, et resta longtemps inexpliquée. 
$>>$

C'est ainsi que j'ai découvert que Mary Tsingou Menzel était toujours en vie et résidait à Los Alamos, à quelques kilomètres seulement de l'endroit où le problème FPU, si important dans le passé et le présent de la physique non linéaire [3], avait été conçu. Il est temps que son travail soit reconnu à sa juste valeur.

\section{Une des premières experts en informatique}

Née le 14 octobre 1928 dans une famille grecque vivant à Milwaukee, Wisconsin, Mary Tsingou a passé sa petite enfance aux États-Unis. En 1936, à cause de la Grande Dépression, sa famille s'installe en Europe, où son père possède une propriété en Bulgarie. Cependant, en juin 1940, suivant les conseils de l'ambassade américaine les incitant par précaution à retourner aux États-Unis, la famille monte dans le dernier navire américain quittant l'Italie. Une semaine seulement après leur débarquement à New York, l'Italie déclara la guerre à la France et au Royaume-Uni.
Tsingou a obtenu sa licence en 1951 à l'Université du Wisconsin et sa maitrise en mathématiques en 1955 à l'Université du Michigan. En 1952, suite à une suggestion de sa professeure d'équations différentielles, elle a posé sa candidature pour un poste au Los Alamos National Laboratory. À l'époque, les femmes n'étaient pas encouragées à poursuivre des études en mathématiques, mais la guerre de Corée avait provoqué une pénurie de jeunes hommes américains, de sorte que des postes étaient également offerts aux jeunes femmes. Elle a donc été embauchée, avec un groupe de jeunes diplômées, pour faire des calculs à la main. Elle a d'abord été affectée à la division T1 de Los Alamos ( $\mathrm{T}$ pour théorique), qui pendant la Seconde Guerre mondiale avait été dirigée par Rudolf Peierls et à laquelle appartenait le célèbre espion Klaus Fuchs. Il y avait à l'étage du dessous la division T7, dirigée par Nicholas Metropolis, qui possédait le tout nouvel ordinateur, le MANIAC I, que quasiment personne ne savait programmer [7]. On proposa à Mary Tsingou, ainsi qu'à d'autres jeunes recrues, d'apprendre justement à l'utiliser. Réussissant au premier essai un exercice qui consistait à coder la fonction sinus, elle impressionna l'instructeur et fut invitée immédiatement à changer de division (et donc d'étage !) pour intégrer le groupe des tout premiers programmeurs. L'ordinateur servait principalement à des tâches liées aux armes mais, de temps en temps et surtout pendant le week-end, les chercheurs pouvaient l'utiliser pour étudier des problèmes de physique et même pour jouer aux échecs. Mary Tsingou et John Pasta ont ainsi été les premiers à créer des graphiques sur l'ordinateur, lorsqu'ils étudièrent ensemble un problème simulant une explosion qu'ils ont pu visualiser sur un oscilloscope.

Mary Tsingou a ainsi interagi avec John Pasta qui fut nommé plus tard à la tête de la National Science Foundation, et aussi avec Stanislaw Ulam, un brillant mathématicien d'origine polonaise, dont plusieurs contributions sur le développement de la bombe à hydrogène ainsi que sur la propulsion nucléaire furent décisives. Elle n'a eu en

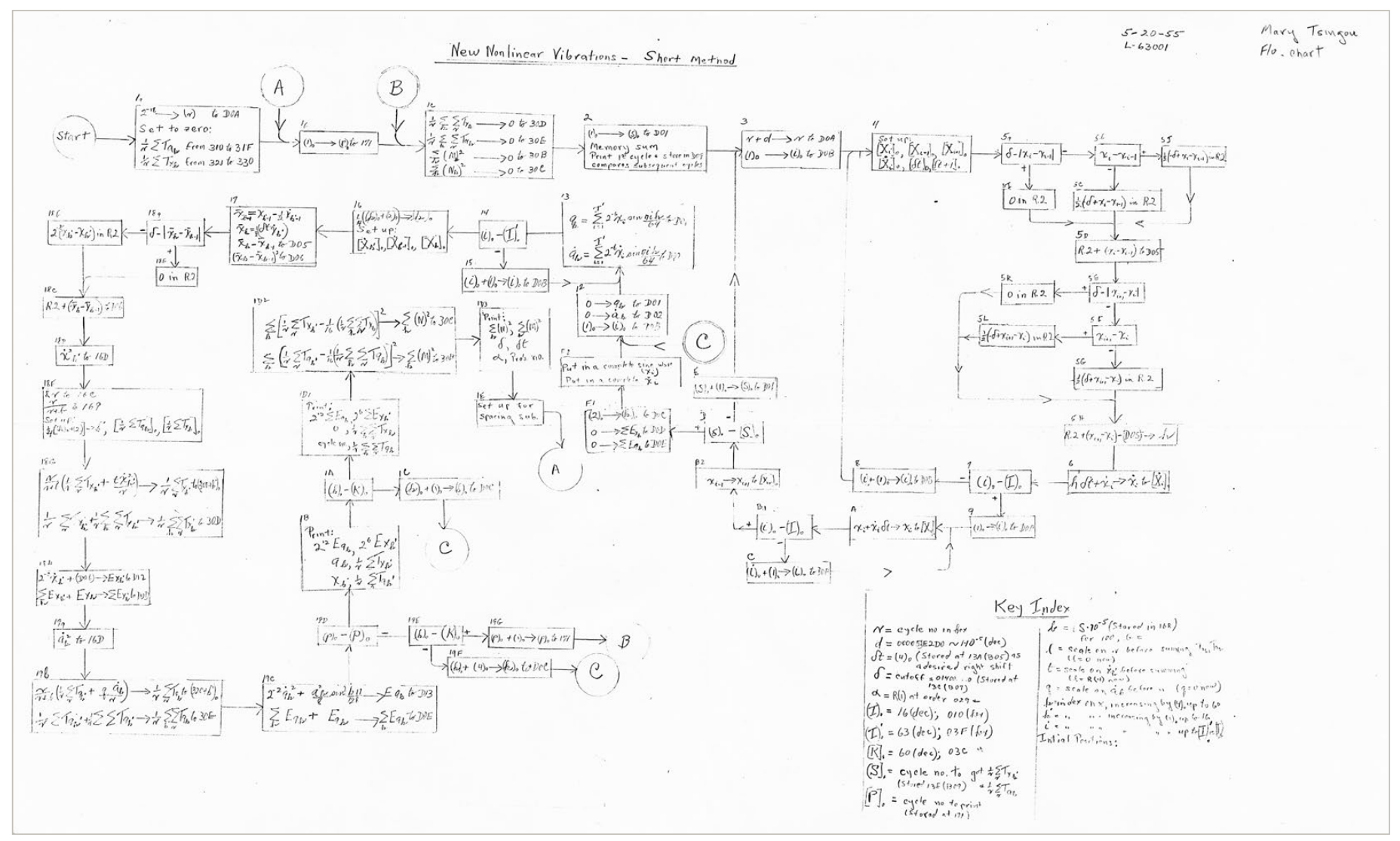

2. L'algorithme utilisé par Mary Tsingou pour coder la première expérience numérique et retrouvé dans sa cave. Notez la date du rapport de Los Alamos, 5-20-55, dans le coin en haut à droite, ainsi que le titre "New Nonlinear Vibrations - Short Method". 
Les phénomènes hors d'équilibre sont au cœur de notre monde

L'expérience numérique de Fermi-Pasta-Ulam-Tsingou (FPUT) [a] peut être considérée comme l'un des points de départ d'un fort engouement pour la physique hors d'équilibre, discipline toujours féconde aujourd'hui. La physique statistique s'est logiquement d'abord intéressée à l'équilibre, qui permet déjà d'expliquer de nombreux phénomènes ; mais le fait même que l'équilibre puisse être atteint n'est pas si évident, même pour des systèmes simples comme l'a montré l'expérience numérique de FPUT. II n'est pas étonnant qu'Enrico Fermi se soit posé cette question puisque, à l'âge de 17 ans, il avait lu puis prolongé certains travaux d'Henri Poincaré, avant de développer à 25 ans la statistique à l'équilibre pour les particules quantiques connues maintenant sous le nom de fermions.

Aujourd'hui encore, cette problématique de la relaxation vers l'équilibre est au cœur de la physique.

1) Il est désormais bien établi que les systèmes physiques avec des interactions à longue portée peuvent avoir des temps de relaxation gigantesques, voire ne pas relaxer vers l'équilibre sur des temps physiquement pertinents [b]. Les systèmes gravitationnels, les fluides géophysiques, les lasers en interaction avec des particules..., font partie de cette catégorie. Cela permet de comprendre pourquoi, alors que les forces gravitationnelles et électromagnétiques sont centrales dans les cours de licence ou de classes préparatoires, elles ne sont pas traitées dans la majorité des cours et livres de mécanique statistique.

2) Les verres forment une deuxième catégorie de systèmes présentant des relaxations vers l'équilibre très lentes [c]. Leur paysage énergétique est extrêmement ramifié et complexe, et explique vraisemblablement leur rigidité. Leurs descriptions dans le cadre de la physique statistique peuvent être aussi considérées comme des modèles pour l'étude des protéines où les questions aussi fondamentales que le désordre, la réponse non linéaire et la relaxation vers l'équilibre sont cruciales.

3) Plus récemment, c'est le monde quantique qui se passionne pour des études tant théoriques qu'expérimentales de cette relaxation vers l'équilibre. De belles expériences [d] consistant à exciter des modes dans des condensats de Bose-Einstein sont même très proches, dans l'esprit, de l'expérience de FPUT.

Étudier tous ces systèmes avec les outils de la physique non linéaire ouvre de nouveaux points de vue très fructueux.

\section{Enseigner la physique des systèmes dynamiques}

Les phénomènes non linéaires sont la règle en physique ! Découvrir à travers un cours introductif les systèmes dynamiques et le chaos devrait faire partie de la formation de tout(e) physicien(ne) et non pas être réservé aux spécialistes. Cette initiation sera essentielle si elle ou il ambitionne d'être chercheur, car la prédominance du concept de déterminisme dans nos formations cache certains comportements pourtant importants. C'est aussi central dans beaucoup d'autres domaines que la physique, par exemple en écologie ou bien en économie, puisque ces deux champs disciplinaires ont mis en évidence que les notions d'instabilité et de bifurcations entre régimes radicalement différents étaient capitales !

Ces idées sont trop peu enseignées dans nos formations et trop peu assimilées par nos concitoyens, même dans les sphères politiques! Comprendre que prévoir la météo pour la fin de la semaine est notoirement difficile (justement les modèles sont chaotiques), mais que cela n'empêche pas de prédire le climat dans quelques dizaines d'années, est important. Cette idée était déjà contenue dans l'excellent article de Edward N. Lorenz (1963) [e] et peut être présentée sans difficulté au niveau Master 1, voire plus tôt en regardant sans modération de splendides et très éducatives vidéos [f]. Lorenz ne s'est pas contenté d'identifier l'essence du chaos (Henri Poincaré l'avait d'ailleurs fait avant lui), en mettant en évidence qu'une description très précise du futur dépend de manière ultra sensible de notre connaissance du présent, beaucoup trop sensible en pratique pour faire des prédictions. Il a aussi, et peut-être surtout, montré qu'une approche statistique permet de prédire des comportements globaux. En effet, le chaos conduit en général à une plus grande exploration de l'espace des phases, ce qui permet d'utiliser la notion de moyenne de façon beaucoup plus efficace. À cause de la sensibilité forte aux conditions initiales, le prévisionniste ne sera donc pas en mesure d'affirmer que « le soleil illuminera le Musée des Confluences à Lyon tel jour précis ». En revanche, il pourrait prédire la température moyenne à Lyon au mois de juillet dans 50 ans. C'est donc l'alliance de la physique non linéaire et de la physique statistique, ce que l'on nomme physique hors d'équilibre, qui se révèle être la bonne approche.

La première expérience numérique codée par Mary Tsingou a donc aussi permis de découvrir un domaine inexploré jusqu'alors et pourtant très important. Au cours des années, la communauté française a joué un rôle prépondérant dans sa compréhension. II faudrait aussi raconter cette histoire.

[a] E. Fermi, J. Pasta, S. Ulam, M. Tsingou, "Los Alamos Scientific Laboratory report LA-1940" (1955). Publié plus tard par E. Segrè (éd.), dans Collected Papers of Enrico Fermi, Vol. 2, U. Chicago Press, Chicago (1965).

[b] A. Campa, T. Dauxois, D Fanelli, S. Ruffo, Physics of Long-Range Interacting Systems, Oxford University Press (2014).

[c] A.P. Young (éd.), Spin glasses and random fields, World Scientific (1998).

[d] N. Navon, A. L. Gaunt, R. P. Smith, Z. Hadzibabic, "Emergence of a turbulent cascade in a quantum gas", Nature 539 (2016) 72-75.

[e] E.N. Lorenz, "Deterministic nonperiodic flow", Journal of the Atmospheric Sciences 20 (1963) 130.

[f] J. Leys, E. Ghys, A. Alvarez : www.chaos-math.org/fr.html. 
revanche que peu de contacts avec Enrico Fermi qui était professeur à Chicago et ne visitait Los Alamos que pendant de courtes périodes durant l'été. Elle connaissait cependant beaucoup mieux la fille de Fermi, Nella, qui ne voulait pas rester en permanence avec ses parents pendant leurs visites à Los Alamos. Les deux jeunes femmes ont donc dormi dans le même dortoir, tandis qu'Enrico et Laura Fermi étaient accueillis par leurs bons amis Stan et Françoise Ulam.

C'est Fermi qui a eu le génie de proposer qu'au lieu de simplement effectuer des calculs standard, les ordinateurs puissent être utilisés pour tester une idée physique. Après ses contributions de toute première importance, tant expérimentales que théoriques, qui lui valent un respect éternel des physiciens, Fermi inventa ainsi le concept d'expériences numériques : c'est beaucoup moins connu. Fermi proposa donc de vérifier la prédiction de la physique statistique sur la thermalisation des solides. Des calculs préliminaires ont confirmé le résultat attendu, à savoir que l'énergie introduite dans un mode de Fourier unique dérive vers d'autres modes. Le comportement quasi périodique n'a pas été observé au début, car l'ordinateur était trop lent pour permettre à une simulation numérique de fonctionner assez longtemps. Mais un jour l'ordinateur ne s'est pas arrêté comme prévu et le calcul a continué de tourner. Les chercheurs ont constaté à leur grande surprise que la quasi-totalité de l'énergie était revenue au mode initial et que l'état initial était presque parfaitement retrouvé. Ce fut le début d'une recherche fructueuse [5].

L'algorithme utilisé par Tsingou sur le MANIAC en 1955 pour simuler la relaxation de l'énergie dans un cristal modèle est reproduit dans la figure 2 ; je l'ai retrouvé en 2007 dans sa cave lors d'une visite chez elle [8]. Sa complexité peut être comparée aux quinze lignes de code Matlab qui sont suffisantes pour une simulation moderne FPU [9]. Programmer les premiers ordinateurs était une tâche qui exigeait beaucoup de perspicacité et d'originalité et, tout au long des années 1960 voire plus tard, les programmeurs étaient souvent mentionnés comme coauteurs. Il a été souvent avancé que la raison pour laquelle Tsingou était citée comme collaboratrice, mais pas comme coauteure, est qu'elle n'avait pas participé à l'écriture du rapport, bien qu'elle ait produit certains graphiques. En fait, Fermi n'était pas impliqué dans l'écriture non plus puisqu'il est mort en 1954, avant la rédaction du rapport [10]. Il me semble plus juste d'avancer que la distinction entre l'écriture et le travail accompli sur le document classé secret a probablement été mal comprise par les lecteurs ultérieurs.

En 1958, Tsingou épousa Joseph Menzel, qui travaillait également à Los Alamos, pour la Force de protection de la Commission de l'énergie atomique. Elle resta dans cette petite ville alors même que ses collègues partaient : Metropolis pour Chicago, Pasta pour Washington et Ulam pour le Colorado. Elle travailla sur de nombreux problèmes différents, toujours avec des ordinateurs. Elle devint l'une des premières expertes en Fortran, inventé par IBM en 1955, et fut chargée d'aider les chercheurs du laboratoire.

Après son travail de programmation sur le MANIAC, Tsingou est revenue sur le problème de FPU avec Tuck au début des années 1970 pour étudier les récurrences plus longues [6]. Elle a également examiné les solutions numériques des équations de Schrödinger et a travaillé avec John von Neumann pour étudier le mélange de deux fluides de densités différentes. Sous la présidence de Ronald Reagan enfin, elle a été aussi profondément impliquée dans les calculs du projet Star Wars.

Retraitée en 1991, Mary Tsingou Menzel vit toujours aujourd'hui à plus de 90 ans avec son mari à Los Alamos, tout près de l'endroit où le problème FPU a été conçu et découvert.

- . . .

On croirait que le nom de Mary Tsingou a été écrit à l'encre sympathique sur l'article fondateur. Il est temps de le faire réapparaitre en reconnaissant sa contribution : parlons donc désormais du problème de Fermi-PastaUlam-Tsingou (FPUT).

\section{Références}

1 E. Fermi, J. Pasta, S. Ulam, M. Tsingou, "Los Alamos Scientific Laboratory report LA-1940" (1955). Publié plus tard par E. Segrè (éditeur), Collected Papers of Enrico Fermi, Vol. 2, U. Chicago Press, Chicago (1965)

2. N. J. Zabusky, M. D. Kruskal, "Interaction of 'Solitons' in a Collisionless Plasma and the Recurrence of Initial States", Physical Review Letters 15 (1965) 240.

3. T. Dauxois, M. Peyrard, Physics of Solitons, Cambridge University Press, New York (2006).

4• F. M. Izrailev, B. V. Chirikov, "Statistical properties of a non-linear string", Soviet Physics-Doklady, 11 (1966) 30.

5. The Fermi-Pasta-Ulam Problem: A Status Report, G. Gallavotti (Ed.), Springer (2008)
6. J. L. Tuck, M. T. Menzel, "The Superperiod of the Nonlinear Weighted String (FermiPasta-Ulam) Problem", Adv. Math. 9 (1972) 399

7. Le MANIAC, pour "Mathematical Analyzer, Numerical Integrator

And Computer", fut construit en 1952 dans le cadre du projet Manhattan et servit notamment à mettre au point la première bombe à hydrogène. Il fut développé à la suite de la frustration de Richard P. Feynman (1918-1987) et Nicholas C. Metropolis (1915-1999), exaspérés par les appareils électromécaniques, lents, bruyants mais pourtant indispensables pour les calculs nécessaires au développement des bombes. Pour la petite histoire, le directeur du projet, Metropolis, aurait choisi le nom MANIAC en espérant mettre fin à la mode naissante de nommer les ordinateurs par des acronymes : cela eut pourtant l'effet totalement inverse ! Cet ordinateur effectuait de l'ordre de $10^{4}$ opérations par seconde environ, à mettre en rapport avec les plus de $10^{9}$ opérations effectuées aujourd'hui par le moindre ordinateur personnel.

8. T. Dauxois, "Fermi, Pasta, Ulam, and a mysterious lady", Physics Today 61, 1 (2008) 55-57.

9. T. Dauxois, M. Peyrard, S. Ruffo, "The Fermi-Pasta-Ulam 'numerical experiment': history and pedagogical perspectives", European Journal of Physics 26 (2005) S3.

10. S. M. Ulam, Adventures of a Mathematician, Scribner, New York (1976). 